Journal of Research in Interprofessional

Practice and

Education

Vol. 5.2

November 2015
Journal of Research in Interprofessional Practice and Education (JRIPE)

Vol. 5.2

(c) 2015

Corresponding author: Xiaoli Gao. Email: gaoxl@hku.hk

\section{Interprofessional Collaboration in Addressing Diet as a Common Risk Factor: A Qualitative Study}

\author{
Hwee Hsia Ong, BDS; Chi Ching Joan Wan, BDS, \& Xiaoli Gao, BDS, PhD
}

\begin{abstract}
Background: Unhealthy diet is a common risk factor threatening dental and general health. Conflicting dietary advice persists among different healthcare professions, despite some shared goals, causing mystification to patients and the public. This qualitative study aimed to understand the perspectives of dentists, physicians, and dietitians in targeting unhealthy diet as a common risk factor, their experiences and barriers in addressing conflicting dietary advice, and possible ways for improving cross-professional coordination.

Methods and Findings: A purposive sample of 40 dentists, physicians, and dieticians was recruited from different service sectors and joined in semi-structured interviews, which were subjected to thematic content analysis. Participants supported the common risk factor approach and suggested improving cross-professional cooperation by maximizing potentials of multidisciplinary care, engaging auxiliary/allied staff, refining electronic systems, and incorporating cutting-edge communication technologies. Inconsistent dietary advice stemmed from different treatment focuses and lack of mutual understanding and well-followed guidelines. Inconsistencies can be resolved by striking a balance for the patient's best interest, well-informing patients, respecting patients' autonomy, acquiring cross-professional knowledge, and conforming to shared guidelines.

Conclusions: Views solicited from three healthcare professions endorsed the importance of cross-professional partnership in preventing/managing dietrelated health problems. Educators, professional bodies, and administrators share the responsibility to dispel conflicting health messages and promote better practice in dietary counselling.

Keywords: Common risk factor; Diet; Health; Interprofessional collaboration; Qualitative research
\end{abstract}

\section{Introduction}

Unhealthy diet has been identified as a common risk factor threatening both oral and general health $[1,2]$. It accounts for many health problems, including cancers, cardiovascular diseases, diabetes, obesity, dental caries, and dental erosion [1]. A balanced diet conducive to general health should be rich in fruit, vegetables, and starchy foods but low in saturated fat, salt, and sugars [3]. From the dental perspective, dental caries is modulated by diet in several ways, mainly the form and frequency of the consumption of sugars and other fermentable carbohydrates, along with the sequence and combination of food intake and foods' potential to stimulate saliva [4]. 
2

Cross-Professional Collaboration in Dietary Advice

Ong, Wan, \& Gao

Journal of Research in Interprofessional Practice and Education

Vol. 5.2

November 2015
Another well-recognized diet-related oral disease is dental erosion. Eating disorders and excessive consumption of citrus fruits and fruit juices have long been implicated in dental erosion [5,6]. Cumulative scientific evidence suggests a lifelong synergy between nutrition and oral health [1]. While a well-functioning oral cavity is necessary for effective mastication and digestion, adequate nutrition is essential for maintaining the integrity of the oral cavity. Given the growing burden of diet-related chronic diseases globally, advocating for a healthy diet has undoubtedly become a focus area for health promotion and patient care [3].

Since unhealthy diet is a risk factor for many chronic diseases, a logical strategy is to adopt a collaborative "common risk factor approach" to health. It is considered more effective and resource efficient and thus preferable to isolated interventions, which merely focus on a specific disease [1,2]. Targeting diet as a common risk factor through cross-professional coordination is being advocated [1]. Nevertheless, there is a lack of consensus on how different professions can collaborate in such efforts.

Physicians, dentists, and dieticians are the three main professions engaged in giving dietary advice. However, despite some shared goals, such as limiting the intake of free sugars, dietary advice provided by the different professions lacks consistency of content, causing mystification to patients and the general public [7]. For instance, "small frequent meals" is advisable from a medical perspective, especially for diabetic and obese patients, whereas dentists suggest combining snacks with main meals to minimize the episodes of acidic challenge on teeth [7]. Dentists do not recommend fruits and juices due to their dental erosive effect; however, they are considered essential for general health [8].

The World Health Organization (WHO), in its recent report, highlighted the importance of interprofessional education and collaborative practice, and suggested some possible mechanisms to achieve them [9]. Multidisciplinary collaboration was also identified as one of the key strategies for promoting healthy diet [3]. In order to better implement the common risk factor approach and overcome the dilemma of cross-professional inconsistencies, a deep understanding of healthcare providers' perspectives needs to be gained. Therefore, this qualitative study aimed to gather the views of three different health professions (physicians, dentists, and dieticians) in targeting diet as a common risk factor, their experiences and barriers in addressing inconsistencies in dietary advice, and their suggestions for possible ways to improve cross-professional coordination.

\section{Method}

A phenomenological stance [10] was adopted in this qualitative inquiry, which aims to understand the views of healthcare providers from three professions. This stance has guided the formulation of research questions and the methodological/analytical choices.

\section{Participant recruitment}

The Institutional Review Board of the University of Hong Kong reviewed the protocol of this study. An ethical approval was obtained (\#UW 14-022). To be eligible to join this study, participants must have fulfilled the following criteria: (a) be a regis- 
3

Cross-Professional Collaboration in Dietary Advice

Ong, Wan, \& Gao

Journal of Research in Interprofessional Practice and Education

Vol. 5.2 November 2015 tered physician or dentist, or accredited dietician in Hong Kong, and (b) be a general practitioner or a specialist in family medicine or family dentistry. Specialists in other fields were excluded. Both genders were included.

A purposive sample of 40 participants was recruited to include three professions (17 physicians, 15 dentists, and 8 dieticians) in different service sectors (17 in public healthcare service, 19 private practitioners, and 4 in university health service). Participants were selected by referring to the lists of registered physicians and dentists and accredited dieticians published on the Web pages of the respective councils and associations, and contacting hospitals and clinics. Details about this study were provided through a participant information sheet. An informed written consent was taken before data collection.

\section{Semi-structured interviews}

Data were collected through face-to-face semi-structured interviews. An interview guide was prepared (see Appendix). A set of open questions were prepared to solicit participants' views and experiences regarding the common risk factor approach, cross-professional inconsistencies in dietary advice, and possible ways for improving interprofessional collaboration.

Each participant was interviewed by one of the three interviewers, who were finalyear dental students trained in the principles and skills of interviewing. The interviewer first explained the aim of the interview and encouraged the participant to speak freely. The participant was also reassured that all opinions collected would be kept confidential and anonymous. Throughout the interview, the interviewer adopted a neutral stance, remained attentive to the participant's responses, and used probing questions to clarify the meanings of responses and invite the participant's elaboration on any relevant issue.

\section{Transcription and thematic analysis}

The audio-recorded interviews were numbered for anonymity, transcribed verbatim, and subjected to thematic analysis [11]. Key concepts were derived inductively. Themes were generated from data and were provisionally tested by the data, without presumptions about the expected content. Line-by-line coding [11] of the interview transcript was carried out manually without using any software. Two final-year dental students analyzed each transcript independently. Both students were trained in thematic analysis methods. In case of disagreement, discussions took place to clarify possible interpretations and modify the codes until a consensus was reached. Representative quotes were selected to illustrate the meaning of the themes and subthemes. To maintain confidentiality, pseudonyms were used in the transcripts and this article. For example, MD-12, DENT-9, and DIET-5 refer to medical doctor \#12, dentist $\#$, and dietician $\# 5$, respectively.

\section{Results}

Participants profile and interview process

Among the 40 participants, there were 24 males and 16 females. Their highest pro- 
JRIPE

4

Cross-Professional Collaboration in Dietary Advice

Ong, Wan, \& Gao

fessional qualifications included a diploma $(n=1)$, bachelor's degrees $(n=22)$, master's degrees $(n=4)$, postgraduate diplomas $(n=5)$, and specialist training in family medicine or family dentistry $(n=8)$. The interviews lasted for 10 to 49 minutes, with an average of 19 minutes. Data saturation was reached after analyzing 34 interviews; thereafter no new themes could be identified.

\section{Common risk factor approach}

Several themes and sub-themes were identified, showing participants' opinions on the common risk factor approach (see Table 1).

Table 1. Common risk factor approach

\begin{tabular}{|l|l|}
\hline Themes & Subthemes \\
\hline Importance of common risk factor approach & Unhealthy diet compromises both general and oral health \\
\cline { 2 - 2 } & Benefits of cross-professional collaboration \\
\hline Means of collaboration & Electronic health system \\
\cline { 2 - 2 } & Multidisciplinary care \\
\cline { 2 - 2 } & Auxiliary and allied health care staff \\
\cline { 2 - 2 } & Diverse communication channels \\
\hline \multirow{3}{*}{ Barriers hindering cooperation } & Constraints of current electronic record system \\
\cline { 2 - 2 } & Isolation in practice and lack of cross-professional contact \\
\cline { 2 - 2 } & Heavy workload \\
\cline { 2 - 2 } & Patients' financial constraint \\
\hline
\end{tabular}

\section{Importance of common risk factor approach}

Interviewees unanimously agreed that unhealthy diet is an important "common risk factor" affecting general and oral health. "Unhealthy diet can compromise patients' health in many aspects. ... Nutritional adequacy is essential for the maintenance of daily metabolism and physiological functions" (DIET-3). Some pointed out the interrelation between diet, general health, and oral health. Examples mentioned include the "two-way relationships between periodontal diseases and diabetes" (DENT-5) and dental pain in "affecting one's appetite, which leads to malnutrition" (DENT-2). Interviewees expressed that collaborations among physicians, dentists, and dieticians on improving patients' diets is essential for quality healthcare. With such a close

Journal of Research in Interprofessional Practice and Education

Vol. 5.2 November 2015 cooperation, a myriad of benefits emerge. Not only does it "promote communication and clustering of knowledge" (MD-10), a tight coordination also allows "better division of labor" and "job specialization" in a team approach (DIET-6). From the patient's prospective, a strengthened coordination contributes to "better quality of patient care and follow up in a long run" (DENT-3). 
5

Cross-Professional Collaboration in Dietary Advice

Ong, Wan, \& Gao

Journal of Research in Interprofessional Practice and Education

Vol. 5.2 November 2015

\section{Means of collaboration}

Coordination among healthcare professions can be facilitated by the electronic patient record system, which helps remove the boundaries between the public and private sectors and can be used as a platform for cross-professional sharing. "Our mode of practice tends to be more computerized.... The consultation notes available in the electronic record do contribute to effective collaboration" (MD-1). The electronic patient record was considered particularly important for managing medically compromised patients since several departments are often involved.

Multidisciplinary care involving a variety of professions, including medical, dental, nursing, and allied health professionals, was considered a helpful way of treating patients with chronic diseases. "The multidisciplinary approach helps improve coordination across different sectors in order to provide a comprehensive and whole-person primary care" (DIET-3).

Interviewees acknowledged the importance of actively engaging auxiliary/allied staff in promoting cross-professional collaboration, since giving dietary advice and monitoring patients progress in diet modification are often delegated to them in both the public and private sectors. "Jobs can be shared among the healthcare team. Dieticians provide dietary advice while nurses keep track of the progress of patients' diet improvement" (MD-3). Auxiliary/allied staff also plays a key role in empowering patients to adopt healthy lifestyles. "Nurses, dieticians, or social workers are also responsible for the patient empowerment program. Through the program, patients are motivated to regain control of and responsibility for their well-being. This longterm behaviour management approach contributes a lot to quality primary care" (MD-1).

Apart from the traditional written referral, other communication channels, such as phone, email, and conference meetings, serve as useful ways to facilitate better communication. "Letter referral is still a formal and courteous way for professional communication. However, for the sake of convenience, making phone calls is sometimes preferred to avoid misunderstanding" (DENT-4). "Multi-specialty conference meetings can facilitate knowledge exchange through case studies or sharing. Cuttingedge techniques can be learned from other disciplines and communication is promoted" (MD-1).

\section{Barriers hindering cooperation}

Despite its wide availability, the electronic system had not yet been fully utilized, as shared by participants from all three professions. "Although medical records were computerized, records were not universally linked and integrated. ... Some units or departments are not equipped with computers, making follow-up difficult" (MD-10). A dentist shared his concerns on the "user-friendliness" of the electronic system. "Although the system allows keeping patients' dental records, it may not be easy to dig out relevant information from the huge volume of medical records" (DENT-11). Another problem with the existing electronic records was the "absence of dietician notes" (MD-8). "We had to ask patients the advice provided by their dietician. The information is indirect and sometimes inaccurate" (MD-1). 
JRIPE

6

Cross-Professional Collaboration in Dietary Advice

Ong, Wan, \& Gao
Journal of Research in Interprofessional Practice and Education

Vol. 5.2 November 2015

\section{Journal of Research in Interprofessional Practice and Education}

The "isolation in practice" and "lack of cross-professional contact" might also "hinder a close cooperation" (DENT-6). An interviewee expressed that the "lack of crossprofessional network hinders prompt referrals to dentists and may compromise patients' oral health" (MD-7). In a multidisciplinary setting such as general hospitals, professionals from different fields work under the same roof and could coordinate as they strive for patients' health. "In a large institution where dental and dietetic units are available, referrals can be simply done via computers. Cooperation can easily be achieved" (MD-16).

Another problem stemmed from participants' heavy workloads. "When work becomes overwhelming, effective communication is obstructed" (MD-10). Last but not least, "patients' financial concern" was brought up as a barrier of cross-professional coordination. "Dietary programs and dental care are not covered in public service and most health insurance plans. Patients often decline referral to a dentist or dietician due to financial concerns" (MD-14).

Inconsistencies in dietary advice

Participants' sharing about the inconsistencies in dietary advice fell into several themes and subthemes (see Table 2).

Table 2. Inconsistencies in dietary advice

\begin{tabular}{|l|l|}
\hline \multirow{3}{*}{ Themes } & Subthemes \\
\hline \multirow{3}{*}{ Causent situation of inconsistencies } & Awareness of the inconsistencies \\
\cline { 2 - 2 } & Lack of coordination \\
\hline \multirow{3}{*}{ Solutions to address inconsistencies } & Different treatment focuses \\
\cline { 2 - 2 } & Lack of mutual understanding \\
\cline { 2 - 2 } & Lack of evidence base and well-followed guidelines a balance for patient's best interest \\
\cline { 2 - 2 } & Well-informing patient and respecting patient autonomy \\
\cline { 2 - 2 } & Enhancing cross-professional understanding \\
\cline { 2 - 2 } & Developing evidence-based guidelines \\
\hline
\end{tabular}

\section{Current situation}

It was not uncommon that interviewees were not too mindful about cross-professional inconsistencies in dietary advice before the interview. They often related this to overspecialization and their limited knowledge about other professions. After hearing some examples, they agreed that such inconsistencies do exist among healthcare professions and all professions need to be alerted. When encountering inconsistencies, they often "avoided commenting on the advice from others," but only "explained their own rationale to patients" (MD-9). 
7

Cross-Professional Collaboration in Dietary Advice

Ong, Wan, \& Gao

Journal of Research in Interprofessional Practice and Education

Vol. 5.2 November 2015

\section{Causes of inconsistencies}

Different treatment focuses in respective expert areas were regarded as the root issue for inconsistencies. "We dentists are concerned about the frequency of sugar intake since it increases risk for dental caries; but doctors and dieticians care more about the effects on the body" (DENT-11). "I think inconsistencies originated from the different diseases and organs we look after. We simply focus on different things" (MD-7).

Interviewees reflected that inconsistencies also stemmed from the lack of mutual understanding. Some doctors and dieticians mentioned that they did not know the dietary advice from dentists and usually did not take patients' oral health into consideration. "We didn't think about the effects of diet on teeth. We just know its effects on the body. Some patients asked if fruit juices cause tooth decay although they are healthy for the body. We suggested they ask their dentists" (MD-7). Some misconceptions on oral health existed among physicians and dieticians, such as "cleaning teeth immediately after eating" (MD-9). Another interviewee asserted "if patients eat more in a meal, the damage to teeth is equivalent to that when separating it into small frequent meals because the total amount is the same" (DIET-2).

The lack of evidence base for some dietary advice was mentioned. "Sometimes information from doctors isn't the most updated. Some patients are told that soya beans increase the risk of breast cancer, but the latest evidence confirmed otherwise" (DIET8). It was mentioned that medical professionals from different areas of practice developed some dietary guidelines; however, dentists' input was not included. "There should be a committee composed of different professions to integrate the evidence-based knowledge of their own fields, so that a shared guideline can be formulated" (DENT-5).

\section{Solutions to address inconsistencies}

Interviewees generally believed that, in the case of inconsistencies, "a balance can be struck without giving up any of the perspectives" (DENT-11), "advice should be given on a case-by-case basis" (MD-8), and the "patient's best interest should always be the top priority" (DENT-3). When a certain dietary plan is needed for treating a health condition but is detrimental to other health aspects, seeking other preventive measures might offset the harmful effects. For example, when small frequent meals are indicated for diabetic patients, other preventive measures such as "proper toothbrushing," "chewing gum," and "fluoride applications" (MD-1) could be considered to compensate for the detrimental effect of such a diet on oral health.

In the case of inconsistencies, patients need to be well informed about all their options and the possible impacts on each aspect of their health. The patient should make the ultimate decision, and the patient's autonomy should be respected. "Discuss with patients about our rationales. They can consider what they want and determine their own priorities. The ultimate decision is out of our hands" (DIET-4).

Mutual understandings and basic knowledge in other professional fields were believed to be essential. "We have to at least know some basics about other professions. With more mutual understanding, we can incorporate other elements for the patient's best interest" (DIET-3). Some interviewees expressed that it is important that dietary advice is "based on solid evidence obtained in clinical research" (DIET-2). It was advo- 
8

Cross-Professional Collaboration in Dietary Advice

Ong, Wan, \& Gao cated that, "a guideline well-supported by evidence from different professions [is] ... generated and made known to professionals and the public" (DENT-5).

\section{Improving cross-professional coordination}

Participants suggested several ways for improving cross-professional collaboration (see Table 3).

\section{Table 3. Improving cross-professional collaboration}

\begin{tabular}{|l|l|}
\hline Themes & Subthemes \\
\hline $\begin{array}{l}\text { Educational institutes and } \\
\text { professional bodies }\end{array}$ & Promoting cross-professional knowledge \\
\cline { 2 - 2 } & Organizing learning activities \\
\hline \multirow{2}{*}{$\begin{array}{l}\text { Health authority and admin- } \\
\text { istrators }\end{array}$} & Developing common guidelines \\
\cline { 2 - 2 } & Establishing Dietetic licensure \\
\cline { 2 - 2 } & Strengthening public primary care \\
\hline
\end{tabular}

\section{Educational institutes and professional bodies}

It was suggested that incorporating elements from other professions could enrich undergraduate curriculums. "If undergraduate students can learn about the different perspectives of various specialties, we can rectify the problem" (MD-11). Measures suggested include "cross-professional lectures" (DIET-4), "joint professional problembased learning" (MD-5), and "cross-professional clinical rotations" (MD-7).

It was believed that professional bodies, such as respective associations, should be proactive in improving cross-professional communication. "Professional bodies can organize joint professional seminars to encourage practitioners to give consistent dietary advice" (MD-9). An interviewee recalled a successful seminar of this kind. "A Chinese medical practitioner gave us a talk on the fundamental principles and treatment rationales for periodontal disease. It strongly promotes cross-professional understandings" (DENT-11). A case-sharing conference was effective in promoting collaboration. "Professional bodies can organize case-sharing conferences. This encourages mutual understandings” (DENT-12).

\section{Health authority and administrators}

Interviewees recommended that a common set of guidelines be developed with input from all healthcare professions. "There should be a committee, which includes doctors, dieticians, and dentists, to integrate evidence-based knowledge from different professions so as to generate a guideline" (DENT-5).

Although dieticians are the experts in diet, their role may not be highly recognized by patients due to the lack of licensing and thus authority. "Self-proclaimed 'nutritionists' may have limited clinical experiences, but dieticians certainly have clinical placement in their curriculum" (DIET-1). It was recommended that the "professional status of dieticians should be raised" (MD-16) by offering licensure in order to facilitate referrals from doctors and dentists. 
9

Cross-Professional Collaboration in Dietary Advice

Ong, Wan, \& Gao

Journal of Research in Interprofessional Practice and Education

Vol. 5.2 November 2015
It was suggested that more resources should be allocated to primary care. "Raising the awareness of healthy diet" and "providing primary dental care to the public" are important to provide well-rounded primary care. "Unless the awareness on healthy diet and the importance of general and oral health are instilled into patients, our collaboration will be in vain" (DENT-9).

\section{Discussion}

This study adopted qualitative research methods, namely semi-structured interviews and thematic content analysis. These methods are exploratory in nature and allow us to probe into related issues and gain some in-depth understandings of the studied topic [12]. A purposive sample of 40 interviewees is considered sufficient for capturing a broad spectrum of views. Specialists, except those in family medicine and family dentistry, were excluded from this study, since the topic may not be so relevant for those practicing in many specialty areas.

Although participants support the importance of the common risk factor approach, it was alarming to hear some interviewees' views that it is rational for different professions to focus on particular diseases and organs. Such a preconception apparently departs from the advocated notion of treating a patient as a whole person [2]. Giving dietary advice without a consideration of patients' total health conditions can be fatal. This was shown in a tragedy in Maryland, where a diabetic patient died after following the advice of a nutritionist who stopped the patient's insulin completely and then stopped her food intake [13]. The WHO has recently embarked on a program that focuses on developing cross-professional inputs for enhancing patient safety and avoiding unnecessary harm [14]. Gathering combined expertise in giving proper dietary advice should be taken as an essential part of this new move.

Interviewees pointed out that physicians and dieticians have limited understanding of the dental perspectives. In the case of inconsistencies in dietary advice, there is a tendency to overlook patients' dental needs. It is possible, however, to maximize patients' general health without compromising their oral health. Diet often takes its effect on oral health through "topical" means, when food and drink pass by or are retained in the oral cavity. Therefore, what matters to dentists is not only what the patients eat or drink, but also how foods and drinks are consumed (e.g., mode and frequency) [15]. If medical professionals are willing to discuss more details with patients, the detrimental effects to their oral health can be minimized [16]. Dentistry is a specialized area of health science. Dental professionals might have to take some initiative to exchange knowledge with other healthcare providers.

On the other hand, physicians and dentists shared that they had inadequate knowledge of nutritional science. This echoes the findings in America, where medical and dental students responded that they had inadequate exposure to nutritional science, which has been underplayed in comparison with other curriculum areas [17]. Although some institutes have incorporated nutritional science as required or elective courses, the number of teaching hours remained low and minimal changes have been seen over the past two decades $[17,18]$. 
10

Cross-Professional Collaboration in Dietary Advice

Ong, Wan, \& Gao

Journal of Research in Interprofessional Practice and Education

Vol. 5.2

November 2015
Modern healthcare has been evolving to meet the demand of complex and fastmoving environments. It is no longer enough for health workers to be professional. They also need to be interprofessional to address the current health challenges [9]. Tertiary institutes remain the main venue to prepare the healthcare workforce for collaborative practice [9]. Multiprofessional learning can be introduced early in undergraduate education, before students become entrenched in conventional professional roles $[19,20]$. It is also important to reinforce this component throughout the curriculum and extend it to residencies, postgraduate studies, and continuing education, so that multiprofessional care is enthusiastically embraced and persistently practiced. Participants' views collected in this study supported that interprofessional education could be more effective if it uses principles of adult learning, if learning methods reflect real-world practice experience, and if interaction occurs in the learning process [9]. By providing opportunities for students to learn about, from, and with each other, they can gain the competency to work individually and as a member of a healthcare team.

While educators and professional bodies share a responsibility in providing training opportunities, health authorities and administrators play a key role in fostering a supportive climate for cross-professional collaboration, establishing mechanisms, and delineating the roles of each party. Through this, experts from different disciplines can form a nucleus, so that a partnership can be developed, strengthened, and expanded. This will help to cut across traditional boundaries and create a synergy among all professions involved in delivering care.

Dietary habits pervade many aspects of people's daily lives and are often not easy to change [21]. Instead of setting unrealistic targets, achievable goals should be negotiable with individual patients, with consideration of his/her health condition, age, and existing lifestyles. Some interim plans may be necessary to help patients achieve their ultimate goal in dietary modification step by step [7]. Exploring possible dietary options with patients under the whole context of general and oral health will possibly empower the patients to take some initial steps toward success.

No two health systems in the world are exactly alike. Nevertheless, as pointed out in the WHO report, experiences gained from other populations might be a useful reference for developing and adapting healthcare policies to meet respective local needs [9]. Views collected in our study may not be directly extrapolatable to other countries. However, our findings shed some light into the perspective of healthcare providers working in different fields and have some implications, especially for many developed countries or regions with similar health education and healthcare systems.

\section{Conclusion}

Views collected from three healthcare professions supported that the common risk factor approach is important in preventing and managing diet-related health problems. Several means can be adopted to coordinate the joint effort from different healthcare professions in addressing diet as a common risk factor. Inconsistency in dietary advice exists among healthcare professions and is a main factor compromising the quality of healthcare. Measures are to be taken to dispel conflicting health messages and promote coherent and comprehensive dietary advice for patients' best 
11

Cross-Professional Collaboration in

Dietary Advice

Ong, Wan, \& Gao

Journal of Research in Interprofessional Practice and Education

Vol. 5.2

November 2015 interests. Educational institutes, professional bodies, and healthcare administrators carry a shared responsibility to promote better cross-professional collaboration in improving the diet of patients and the general public.

\section{Acknowledgements}

The authors are grateful to all interviewees for their participation as well as the support of The University of Hong Kong Research Output Prize. The participant recruitment, data collection, and data analysis were completed through the group effort of undergraduate dental students Hiu Yeung Lai, Wing Ho Man, Chun Kit Mo, On Kei Angela Poon, Ching Yan Tam, Kwan Lok Tse, and Karissa Joyce Yip.

\section{References}

1. Academy of Nutrition and Dietetics. (2013). Position of the Academy of Nutrition and Dietetics: Oral health and nutrition. Journal of the Academy of Nutrition and Dietetics, 113, 693-701.

2. Sheiham, Aubrey, \& Watt, Richard G. (2000). The common risk factor approach: A rational basis for promoting oral health. Community Dentistry and Oral Epidemiology, 28, 399-406.

3. World Health Organization. (2004). Global strategy on diet, physical activity and health. URL: http://www.who.int/dietphysicalactivity/strategy/eb11344/strategy_english_web.pdf [May 3, 2015].

4. Konig, Klaus G. (2000). Diet and oral health. International Dental Journal, 50, 162-174.

5. Studen-Pavlovich, Deborah, \& Elliott, Margaret A. (2001) Eating disorders in women's oral health. Dental Clinics of North America, 45, 491-511.

6. Moynihan, Paula J. (2002). Dietary advice in dental practice. British Dental Journal, 193, 563-568.

7. Armstrong, Alison, Freeman, Ruth, McComb, Angela, Speedy, Patti (2008). Nutrition and dental health: Guidelines for professionals. URL: http://www.healthpromotionagency.org.uk/resources /nutrition/pdfs/nutrition_and_dental_health.pdf [October 28, 2015].

8. Committee on Nutrition. (2001). The use and misuse of fruit juice in pediatrics. Pediatrics, 107, 1210-1213.

9. World Health Organization. (2010). Framework for action on interprofessional education and collaborative practice. URL: http://www.who.int/hrh/resources/framework_action/en/ [May 3, 2015].

10. Giorgi, Amedeo. (1997). The theory, practice, and evaluation of the phenomenological method as a qualitative research procedure. Journal of Phenomenological Psychology, 28(2), 235-260.

11. Braun, Virginia, \& Clarke, Victoria. (2006). Using thematic analysis in psychology. Qualitative Research in Psychology, 3(2), 77-101.

12. Bower, Elizabeth, \& Scambler, Sasha. (2007). The contributions of qualitative research towards dental public health practice. Community Dentistry and Oral Epidemiology, 35, 161-169.

13. Zanteson, Lori. (2012). RD licensing legislation-Is it a matter of public safety or monopoly? Today's Dietitian, 14, 46.

14. World Health Organization. (2011). Patient safety curriculum guide: Multi-professional edition. URL: http://apps.who.int/iris/bitstream/10665/44641/1/9789241501958_eng.pdf [October 28, 2015].

15. Moynihan, Paula, \& Petersen, Poul Erik. (2004). Diet, nutrition and the prevention of dental diseases. Public Health Nutrition, 7, 201-226.

16. Bassiouny, Mohamed A., \& Yang, Jie. (2005). Influence of drinking patterns of carbonated beverages on dental erosion. General Dentistry, 53, 205-210.

17. Touger-Decker, Riva. (2004). Nutrition education of medical and dental students: Innovation through curriculum integration 1-4. American Journal of Clinical Nutrition, 79, 198-203.

18. Romito, Laura M. (2003). The prevalence of dietary counseling activities in North American dental schools. Journal of Dental Education, 67, 206 (abstract 91).

19. Lindqvist, Susanne, Duncan, Anna, Shepstone, Lee, Watts, Fiona, \& Pearce, Shirley. (2005). Casebased learning in cross-professional groups-The development of a pre-registration interprofessional learning programme. Journal of Interprofessional Care, 19, 509-520.

20. World Health Organization. (1988). Learning together to work together for health. Report of a WHO study group on multiprofessional education of health personnel: The team approach. World Health Organization Technical Report Services, 769, 1-72.

21. Rollnick, Stephen, Mason, Pip, \& Butler, Chris. (1999). Health behavior change: A guide for practitioners. London, UK: Churchill Livingstone. 


\section{JRIPE}

Cross-Professional Collaboration in Dietary Advice

Ong, Wan, \& Gao
Journal of Research in Interprofessional Practice and Education

\author{
Appendix \\ Interview guide
}

\section{A. Common risk factor}

1. In your opinion, how important is it (and why) for different professions to work together on diet for improving patients' health?

2. What are the possible ways for different professions to coordinate in improving patients' diets and their overall health?

3. Would you expect any barriers in such coordination?

\section{B. Inconsistencies}

4. What do you think about these types of inconsistencies?

5. Could you share with us any patient cases that were troubled with this kind of inconsistent advice?

i. What inconsistent advice did the patients receive?

ii. What did the patients feel?

ii. What did you suggest to him/her?

6. Knowing that such inconsistencies exist, how do you think that different professions can balance their dietary advice in the best interests of their patients?

7. Would you imagine any difficulties in balancing the different dietary advice?

\section{Recommendations}

8. How do you think the curriculum can be improved/integrated so that graduates can be more prepared to coordinate across professions?

9. How can the government, authorities, and regulatory bodies help coordination across professions?

10. Any other questions/opinions that you want to share? 